\title{
New diagnosed heart failure with renal insufficiency - Cardiac MRI-guided clinical decision making
}

\author{
Calin Maniu ${ }^{1}$ and Kana Fujikura ${ }^{2}$ \\ ${ }^{1}$ Johns Hopkins Medicine \\ ${ }^{2}$ National Institutes of Health
}

March 24, 2021

\begin{abstract}
A 63 year-old female with multiple atherosclerotic risk factors presented with newly diagnosed heart failure. Clinical presentation and initial work-up studies (e.g. laboratory findings, ECG, and echocardiography) were indeterminate for ischemic or non-ischemic etiology. As she developed contrast-induced nephropathy, coronary angiography was deferred and cardiac MRI was performed instead. Cardiac MRI elegantly demonstrated non-ischemic cardiomyopathy that was subsequently confirmed by invasive coronary angiography. This case emphasizes the important role of cardiac MRI in establishing the etiology of cardiomyopathy, ultimately altering the clinical management of the patient with newly diagnosed heart failure.
\end{abstract}

\section{Title Page}

New diagnosed heart failure with renal insufficiency - Cardiac MRI-guided clinical decision making

\section{Running Head}

MRI-based etiology assessment in cardiomyopathy

\section{Authors}

Calin V. Maniu, MD

Cardiology, Johns Hopkins Community Physicians - Heart Care

8600 Old Georgetown Rd

Bethesda, MD

FAX: 4103672059

Kana Fujikura, MD, PhD

Advanced Cardiovascular Imaging Laboratory, National Heart, Lung and Blood Institute,

National Institutes of Health, Department of Health and Human Services.

10 Center Drive, Bldg 10, Rm B1D416

Bethesda, MD 20892-1061

FAX: none

\section{Corresponding Author}

Kana Fujikura, MD, PhD 
Advanced Cardiovascular Imaging Laboratory, National Heart, Lung and Blood Institute,

National Institutes of Health, Department of Health and Human Services.

10 Center Drive, Bldg 10, Rm B1D416

Bethesda, MD 20892-1061

Kana.fujikura@nih.gov

Phone: 301-503-8733

FAX: none

\section{Author contribution}

Calin V. Maniu - patient's clinical management, echocardiography, drafting article, Critical revision of article

Kana Fujikura - cardiac MRI, drafting article, Critical revision of article

\section{Key Words}

Heart failure, cardiac magnetic resonance imaging, ischemic cardiomyopathy, cardiomyopathy

Funding: none

Disclosures: There are no relationships with industry.

\section{Abstract}

A 63 year-old female with multiple atherosclerotic risk factors presented with newly diagnosed heart failure. Clinical presentation and initial work-up studies (e.g. laboratory findings, ECG, and echocardiography) were indeterminate for ischemic or non-ischemic etiology. As she developed contrast-induced nephropathy, coronary angiography was deferred and cardiac MRI was performed instead. Cardiac MRI elegantly demonstrated non-ischemic cardiomyopathy that was subsequently confirmed by invasive coronary angiography. This case emphasizes the important role of cardiac MRI in establishing the etiology of cardiomyopathy, ultimately altering the clinical management of the patient with newly diagnosed heart failure.

A 63 year-old female with hypertension, hypercholesterolemia and active cigarette smoking presented with orthopnea and dyspnea on exertion that had worsened over the previous 2 months. Pulmonary embolism was ruled out with chest CT angiogram. ECG showed new T-wave inversion in the lateral wall when compared to an old tracing (Figure 1). Laboratory data showed severely elevated pro-BNP $(23,615 \mathrm{pg} / \mathrm{mL})$. Initial troponin $\mathrm{T}$ was undetectable. Her renal function worsened secondary to contrast-induced nephropathy $(\mathrm{Cr}$ $1.5 \mathrm{mg} / \mathrm{dL}$ [baseline $0.9 \mathrm{mg} / \mathrm{dL}$ ], eGFR $42 \mathrm{ml} / \mathrm{min} / 1.73 \mathrm{~m}^{2}$ [baseline $71 \mathrm{ml} / \mathrm{min} / 1.73 \mathrm{~m}^{2}$ ]). Echocardiography showed biventricular dilatation and decreased systolic function with regional wall motion abnormalities (Video 1). As the etiology of the cardiomyopathy was not clear, CMR was performed for further evaluation.

Cardiac MRI cine imaging showed severely dilated LV with severely decreased systolic function (LVEF 17\%) with global hypokinesis (Video 2). On late gadolinium enhancement imaging, there was patchy myocardial fibrosis in the anterolateral papillary muscle and a small portion of the mid lateral wall, mid-wall fibrosis in the basal anteroseptal segment, and fibrosis at both anterior and inferior right ventricular insertion sites (Figure 2A). Cardiac MRI findings were compatible with non-ischemic cardiomyopathy. Invasive coronary angiography performed subsequently revealed normal coronaries (Figure 2B). Antiplatelet therapy was discontinued. She was discharged home with low dose furosemide, carvedilol, losartan, isosorbide dinitrate, and hydralazine. Sacubitril/valsartan was subsequently initiated as an outpatient, once renal function stability has been confirmed.

Coronary artery disease is the most common cause of systolic heart failure in Western countries. Ischemic cardiomyopathy is associated with decreased survival when compared to non-ischemic cardiomyopathy (1). If feasible, surgical coronary revascularization improves survival when compared to medical management 
(2). This case emphasizes the important role of cardiac MRI in establishing the etiology of cardiomyopathy, ultimately altering the clinical management of the patient with newly diagnosed heart failure.

\section{Figure Legends:}

Figure 1. ECG on admission.

Figure 2. (A) Cardiac MRI: late gadolinium enhancement imaging (PSIR) in short axis stack. There is patchy myocardial fibrosis in the anterolateral papillary muscle and a small portion of the mid lateral wall (thick arrows). There is a mid-wall fibrosis in the basal anteroseptal segment (arrow head). Additionally, there is fibrosis at both anterior and inferior right ventricular insertion sites (thin arrows). The pattern of myocardial fibrosis is compatible with non-ischemic cardiomyopathy. (B) Coronary angiography: (left) RAO cranial of LCA, (middle) LAO caudal view of LCA, and (right) LAO view of RCA. There was no significant coronary artery disease.

Video 1. Echocardiography of apical (A) 4-chamber, (B) 2-chamber, and (C) 3-chamber views. The left ventricle was mildly dilated with severely decreased function. The right ventricular function was moderately decreased. The left ventricular wall motion was globally severely hypokinetic with regional variations. Inferior and inferoseptal segments were akinetic, whereas the wall motion of basal anterior and lateral segments were rather preserved. The findings were suggestive of ischemic cardiomyopathy.

Video 2. Cardiac MRI steady-state free precession (SSFP) cine imaging of long axis views: (A) 2-chamber, (B) 3-chamber, and (C) 4-chamber views. Cardiac MRI cine imaging showed severely dilated left ventricle with severely decreased systolic function (ejection fraction 17\%). The left ventricular wall is globally severely hypokinetic with minimal regional variations. There were prominent trabeculations in the anterior wall from base to apex, that did not meet the criteria for non-compaction. The right ventricle was severely dilated with moderately decreased systolic function.

\section{References}

1. Felker GM., Shaw LK., O'Connor CM. A standardized definition of ischemic cardiomyopathy for use in clinical research. J Am Coll Cardiol 2002;39(2):210-8.

2. Wolff G., Dimitroulis D., Andreotti F., et al. Survival benefits of invasive versus conservative strategies in heart failure in patients with reduced ejection fraction and coronary artery disease: A meta-analysis. Circ Hear Fail 2017;10(1):e003255.

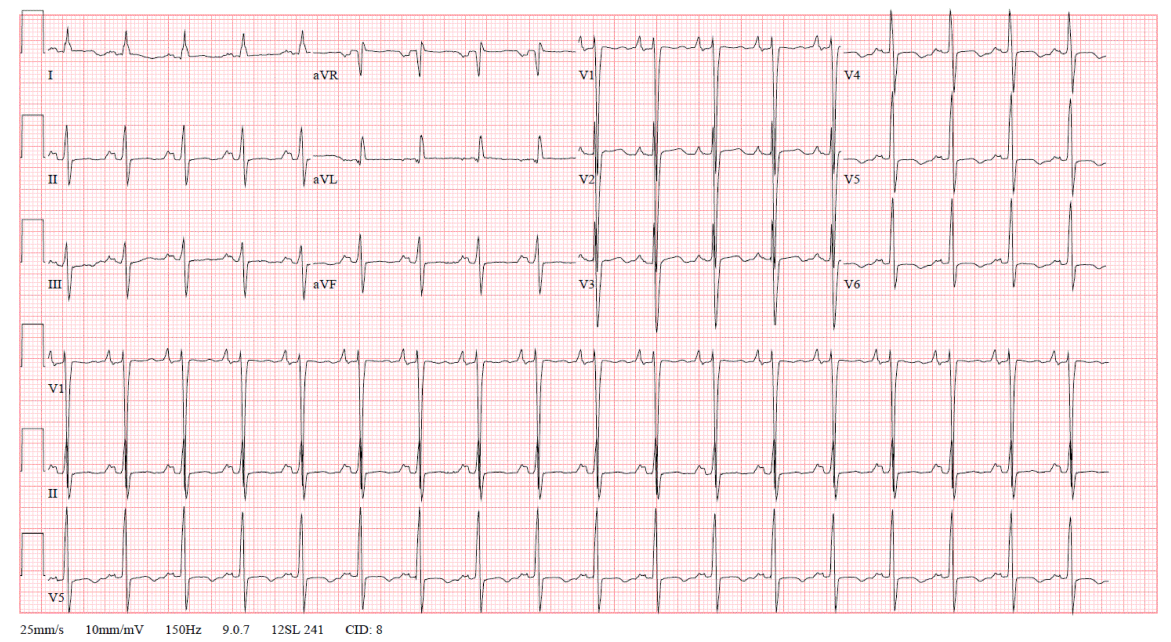


(A)

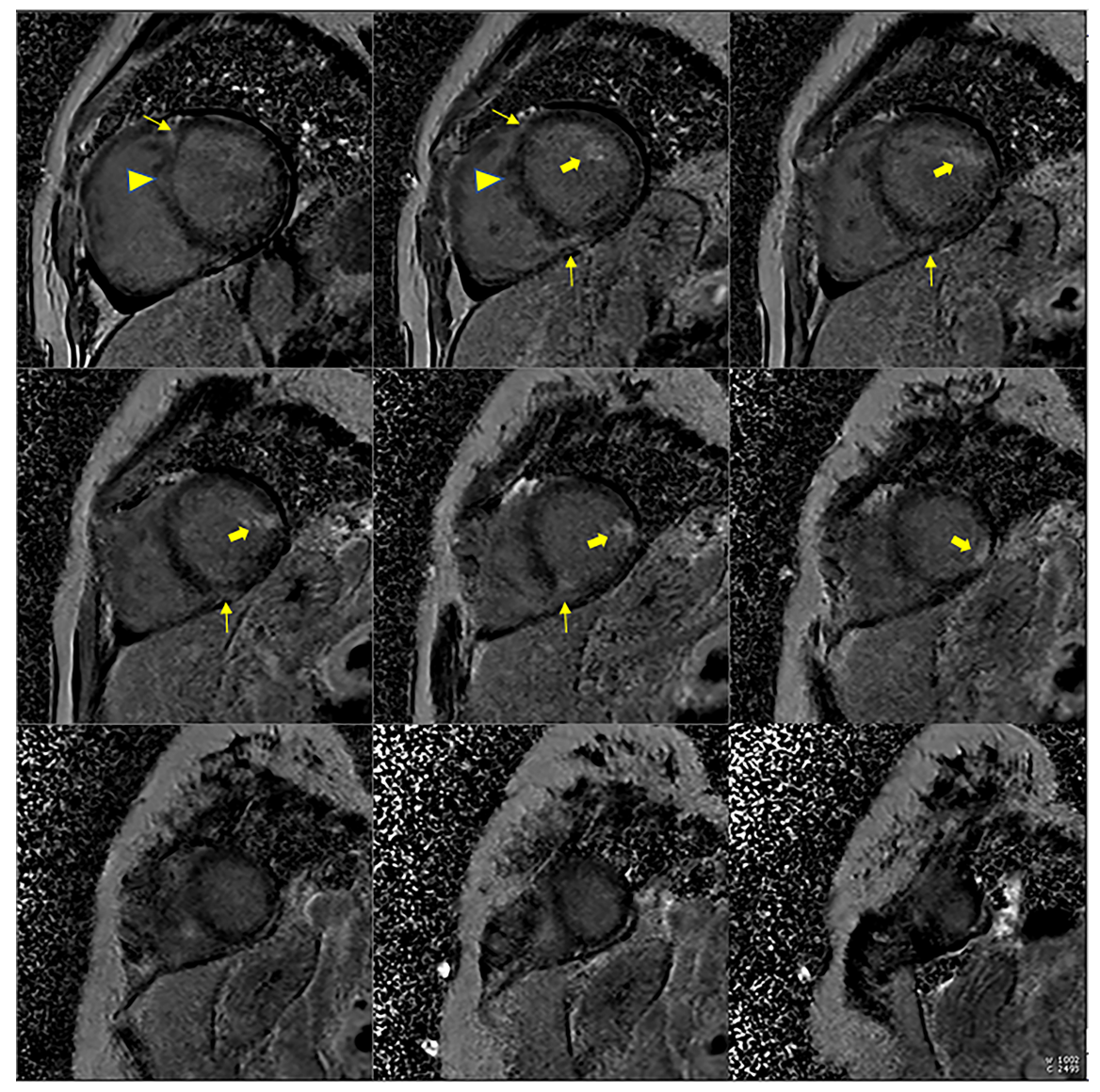

(B)

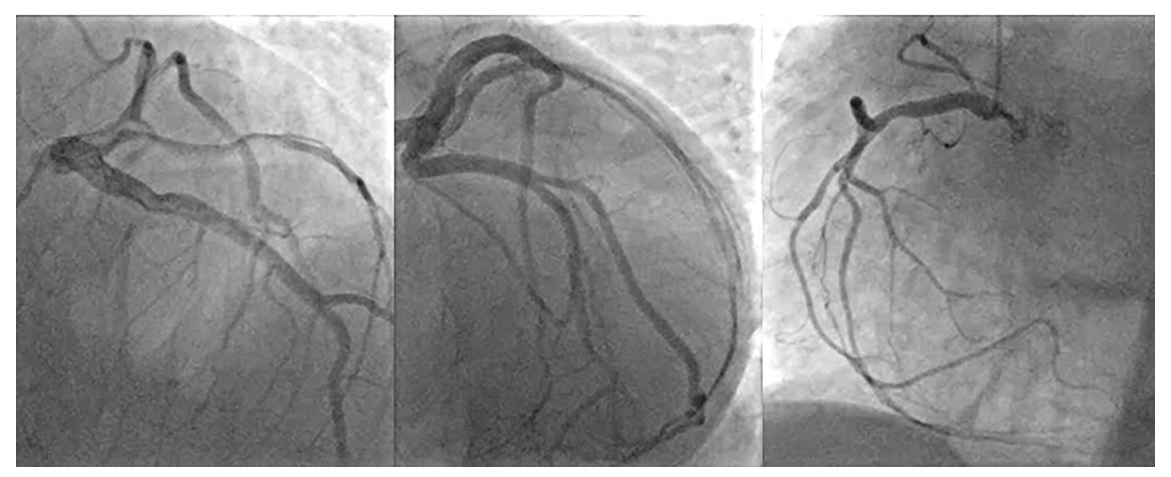

